\title{
One step closer to personalized therapy with cell capturing
}

A major challenge in treating metastatic prostate cancer is that secondary tumors, and the circulating cells that seed them, are fundamentally different to the primary lesion from which they are thought to originate. In the current era of intense research into personalized therapy, understanding the underlying biology of the metastases is as important as that of primary tumor. Scientists have now shown that circulating tumor cells can be captured from the blood quickly and easily so they can be studied, placing us one step closer to practical individualized cell analysis and, ultimately, therapy.

The method is based on capturing prostate-specific membrane antigen (PSMA) - expressed by all prostate cancer cells-with the small-molecule inhibitor CTT-554. The inhibitor is conjugated to biotin, which has a strong affinity for the protein streptavidin. Once the blood sample has been treated with the inhibitor, small magnetic beads ( $\sim 1 \mu \mathrm{m}$ in diameter) coated with streptavidin bind to the PSMA-CTT-554-biotin complex on the surface of the cells. "Applying a magnet to the sample traps the prostate cancer cells against the wall of the sample tube," lead investigator Cliff Berkman explained. The other cells in the blood sample can be washed away, leaving only the prostate cancer cells. Removing the magnet releases the captured cells, which remain viable. The whole process takes only a few hours.

Using this innovative procedure, up to $97 \%$ of cancerous cells were captured from a blood sample spiked with LNCaP cancer cells, with $92 \%$ of these remaining viable. However, the capture efficiency is concentration-dependent and decreases with lower levels of cancer cells in the blood. Regardless, at the lowest limits of capture enough viable cells could be captured for further study.

The power of the tool is most evident when considering the possibility that the

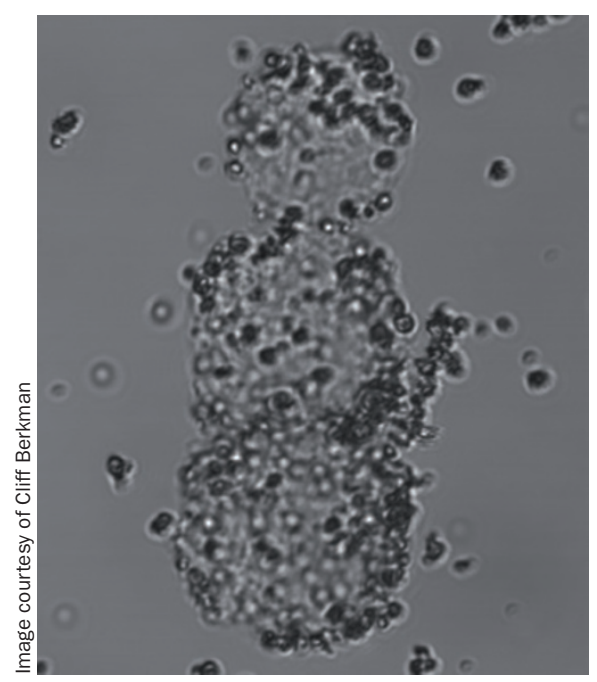

captured tumor cells could subsequently be propagated in vitro. "While much is known about primary and metastatic prostate tumors, very little is known about these circulating tumor cells in terms of their abundance, expression levels of cell-surface biomarkers, metastatic potential and responsiveness to therapy," says Berkman. If suitable conditions can be determined for their culture, the cells could be used to develop in vitro models to further understand their relevance in the progression of the disease. Importantly, if these cells are indeed representative of those that ultimately develop metastases, various chemotherapeutic drugs can be tested to determine optimal prostate cancer therapy for each patient using their own cells. Berkman and his team are now beginning to work with clinical investigators to implement this technology on blood samples from patients with prostate cancer.

Mina Razzak

Original article Wu, L. W. et al. Chemoaffinity capture of pre-targeted prostate cancer cells with magnetic beads. Prostate doi:10.1002/pros. 225088 